\title{
26 DEVELOPMENT OF A RECORD LINKAGE SYSTEM TO SUPPORT INJURY SURVEILLANCE AND THE EVALUATION OF INTERVENTIONS
}

doi:10.1136/injuryprev-2012-040580b.26

RA Lyons*, S Macey, S Turner, DV Ford. Swansea University, UK

Background Observational and interventional epidemiology have developed apart with separate data systems and skill sets. There is a need to bring these together to speed translation of knowledge to support developing and testing new interventions, and widespread adoption of those which are effective to maximise population benefit.

Aims/Objectives/Purpose To develop an integrated population based information system to support injury prevention and control.

Methods The Secure Anonymised Information Linkage (SAIL) system was created for the population of Wales (approximately 3 million individuals) from multiple datasets. Anonymisation occurs at individual and household level. Birth, migration and death data are linked to inpatient, outpatient, emergency department (ED), general practice (GP) and other datasets. 
Results/Outcomes During the period 1 April 2009 to 31 March 2010 the number of injured individuals within the ED, inpatient and mortality datasets totalled 330183 ; 42610 and 1102 respectively. These cases can be linked to birth, death and residential records from the Welsh Demographic Service, a dataset comprising information on approximately 4.5 million living and dead people in Wales. Linkages can also be made to approximately 1.4 million anonymised households plus a growing GP dataset currently encompassing $40 \%$ of the GPs across Wales. Evaluations underway include: targeting of house fire prevention, home improvements, the impact of energy efficiency interventions, and the impact of changing access to alcohol outlets.

Significance/Contribution to the Field The SAIL system represents a significant development in capability to evaluate injury prevention and control interventions and policies whilst also undertaking surveillance. 